\title{
O ESTATUTO ATUAL DA LÍNGUA INGLESA: COMO ALUNOS- PROFESSORES DO CURSO DE LETRAS COMPREENDEM E SE IDENTIFICAM COM AS DIFERENTES PERSPECTIVAS DE USO E ENSINO DO IDIOMA
}

\author{
Taisa Pinetti PASSONI \\ Juliane D'ALMAS
}

Luciana Cristina da Costa AUDI

\begin{abstract}
Resumo
O presente trabalho visa apresentar como os alunos-professores do terceiro ano do curso de Letras Estrangeiras Modernas-Inglês da Universidade Estadual de Londrina compreendem diferentes perspectivas para uso e ensino de Língua Inglesa no mundo contemporâneo e com qual delas mais se identificam ao ensinar o idioma. Após leituras e discussão a respeito dos diferentes acrônimos e suas respectivas conceitualizações para se referir ao Inglês (EFL, ESL, EIL, ELF, WE) os alunos-professores postaram comentários em um blog relatando suas representações a respeito. Através das análises destes comentários, compreendemos que este assunto é ainda muito novo no processo de formação destes alunos-professores, o que nos sugere algumas mudanças que poderão ser realizadas a fim de que estes possam se profissionalizar atingindo uma melhor compreensão de seu papel como professores de Língua Inglesa na sociedade.
\end{abstract}

Palavras-chave: formação de professores; estatuto da Língua inglesa; ensino; uso.

\section{Abstract}

The current paper aims to present how third-year student-teachers of English from the Language Arts Course - Modern Foreign Languages- at Universidade Estadual de Londrina understand different perspectives for the use and the teaching of the English Language in the contemporary world. It also aims to show with which of those perspectives they most identify themselves as they teach this language. After reading and discussing about different acronyms and their respective conceptualizations in order to refer to English Language (EFL, ESL, EIL, ELF, WE), the student-teachers posted 
comments in a blog presenting their representations about the topic. Through analyses of these comments, we understand that this subject is still brand new in the process of teachers' education, what suggests some changes which can be made so that they can professionalize themselves achieving a better comprehension about their role as English Language teachers in the society.

Keywords: teacher education; English language status; teaching; use.

\section{A Língua Inglesa na contemporaneidade}

Estamos em meio a mudanças sociais de um modelo modernista de globalização - que estratifica comunidades e linguagens de modo hierárquico -para um modelo mais fluido de globalização pós-moderna. Essa realidade traz profundas implicações para o ensino e o uso da Língua Inglesa, já que este idioma tem um papel importante nas relações entre as culturas, ao mesmo tempo que possibilita os fluxos globais (CANAGARAJAH, 2005).

O Inglês tem sido percebido como uma fonte indispensável para a inserção econômica em âmbito mundial, razão pela qual muitos governos o vêem como alavanca de desenvolvimento em diversos âmbitos, capaz de promover modernização material e tecnológica. Por essa razão, investem no seu aprendizado como forma de capital humano (LIN \& MARTIN, 2005).

De tal contexto deriva a realidade atual da Língua Inglesa. Seu caráter vem se modificando: deixa de ser apenas a Língua Materna dos países do Círculo Interno ${ }^{1}$ e passa a atingir uma comunidade global, a qual fala diferentes variedades com diferentes normas, onde as pessoas têm que negociar essa diversidade por onde forem. De acordo com Canagarajah (2005):

O novo papel do inglês significa que nós devemos ter uma orientação diferente em relação à língua. As variedades pertencentes às diferentes comunidades - tanto as tradicionais 'variedades nativas' quanto variedades 'não-nativas' - ganharam importância uma vez que o

\footnotetext{
${ }^{1}$ KACHRU, 1989 apud MCKAY, 2002.
} 
Inglês serve de suporte comum para se realizar um trabalho nas fronteiras. ${ }^{2}$ (p. 23)

Assim, seria necessário deixar para trás o desejo de um sistema gramatical uniforme e desenvolver estratégias pragmáticas para negociar o pluralismo lingüístico a fim de preparar o cidadão, enquanto estudante, para a sociedade do conhecimento. $\mathrm{O}$ autor, no entanto, nos alerta para o fato de que essa abordagem é considerada confusa por alguns acadêmicos, por considerarem que esse multilinguismo poderia causar atritos e desunião. Uma tentativa de evitar tais desajustes seria um esforço da sociedade, principalmente dos professores de Língua Inglesa, para desenvolver práticas que estimulem a diversidade linguística.

Diante deste cenário surge uma inquietante dúvida para os professores de Língua Inglesa: que Inglês é esse que estamos ensinando? As origens do ensino de inglês como Língua Estrangeira (LE) nos remetem a contextos limitados e até mesmo elitistas, os quais privilegiavam as classes sociais de maior poder aquisitivo. Embora atualmente perspectivas concorrentes sejam concomitantes, o foco está voltado para o uso que essa LE terá fora dos domínios da sala de aula, em um contexto mais amplo, onde se coloca em xeque os limites das relações entre a língua e seu pertencimento a determinado país e/ou cultura.

Dada a realidade com que nos deparamos, torna-se premente que nos cursos de formação de professores levantem-se discussões a este respeito, a fim de se promover coerência entre o Inglês ensinado na sala de aula e aquele que o cidadão poderá realmente utilizar como forma de comunicação. Por essa razão, diante das mudanças econômicas e da expansão da Língua Inglesa com a globalização, diversos autores demonstram-se preocupados com toda essa problemática. Na verdade, começaram, em seus estudos, a abordar as diferentes terminologias pelas quais o Inglês passou a ser definido, levando-se em conta seus diferentes estatutos ao redor do mundo.

Autores como Seidlhofer (2001), Graddol (2006), Dewey (2007), Pakir (2009) entre outros, defendem a escolha do Inglês como a Língua Franca (ELF) ${ }^{4}$. Para Graddol, o principal requisito para o ensino de ELF seria a inteligibilidade, razão pela

\footnotetext{
2 "The new role of English has meant that we have to orientate to the language differently. The varieties belonging to the different communities - both the traditional 'native varieties' and 'non-native' varieties have gained importance as English serves as the common medium for getting work done across borders." 3 Todas as traduções que constam neste artigo foram livremente feitas pelas autoras.

${ }^{4}$ Optamos por manter a forma original das siglas referentes às terminologias em inglês, porque tratam-se de jargões da área e raramente são traduzidas.
} 
qual ensinar algumas características de pronúncia poderia ser considerado "perda de tempo". A perspectiva de ELF foca ainda nas estratégias pragmáticas requeridas para a comunicação internacional, de modo que o modelo-alvo do inglês não é o do falante nativo, mas a do falante bilíngue fluente, o qual conserva uma identidade nacional em termos de sotaque e possui habilidades especiais necessárias para negociar a compreensão com outro falante não-nativo.

Pensar em ELF traz implicações pedagógicas, i.e., a necessidade de pensar o ensino desse idioma não apenas como sistema e uso. De acordo com essa perspectiva, ser um usuário competente do Inglês significa ter uma consciência crítica e linguística sobre que peso tem a língua que se fala.

No que concerne o EIL, inglês como uma língua internacional, Pennycook (1994), ao discutir a expansão da Língua Inglesa, questiona o discurso circulante sobre tal estatuto do inglês bem como suas implicações culturais e políticas conforme têm sido entendidas. Segundo o autor, imaginar que a expansão dessa língua pode ser considerada natural, neutra e benéfica é um equívoco. Para os que advogam tais características e são por ele criticados, a referida expansão seria natural porque apesar das críticas ao inglês colonial, dos falantes terem sido colônias da Inglaterra, a língua é também o resultado inevitável da globalização; neutra porque torna-se independente do seu contexto cultural, seja americano ou britânico, não apresenta ameaças, sendo um meio neutro e transparente de comunicação; e benéfica a partir do momento que é valorizada tanto como língua de comércio, ciência e tecnologia, quanto é reconhecida como uma língua internacional para comunicação, uma vez que é amplamente utilizada.

Ainda na perspectiva do EIL, Gimenez (2008) afirma que esta terminologia implica assumir a existência de uma única variedade que seria adotada internacionalmente. Já para Trudgill E Hannah (1995 apud PAKIR, 2009) o EIL é:

A variedade da Língua Inglesa, que é normalmente empregada na escrita e normalmente falada pelos falantes "cultos" da língua. É também, com certeza, a variedade do Inglês que estudantes de Língua Estrangeira ou Segunda Língua (EFL/ESL) aprendem quando recebem instrução formal. ${ }^{5}$ (p. 225)

\footnotetext{
5 The variety of English language which is normally employed in writing and normally spoken by "educated" speakers of the language. It is also of course, the variety of English that students as Foreign or Second Language (EFL/ESL) are taught when receiving formal instruction.
} 
Isto é, para os autores, o EIL seria ensinado àqueles que aprendem um idioma de forma sistemática e formal, na escola ou em institutos de línguas. Utilizam, ainda, duas outras terminologias para melhor explicar o EIL. Falam sobre Inglês como Língua Estrangeira (EFL) e do Inglês como Segunda Língua (ESL), o que de acordo com os estudos de Graddol (2006) seria algo incoerente, já que, para ele, trata-se de coisas distintas.

Graddol (2006) trata do acrônimo EFL como um dos primeiros a se referir a uma das abordagens mais conhecidas para o ensino de Inglês. Essa perspectiva enfoca a importância da eficácia do aprendizado centrado na imitação do falante nativo, não apenas em relação ao domínio da língua, mas também no que diz respeito a aspectos culturais e sociais. Numa crítica a este modelo, o autor aponta que o aprendiz assume a posição de estrangeiro, de turista linguístico, que luta para ser aceito pela comunidade da língua-alvo.

O mesmo autor define também o ESL, um modelo contrastante com o citado anteriormente. A principal característica desta perspectiva é que ela reconhece o papel do Inglês na comunidade em que ele é ensinado. Segundo Graddol (Ibid.), historicamente surgiu da necessidade do Império Britânico de ensinar a língua para a população local em suas colônias a fim de que, nelas, eles pudessem administrar órgãos da corte. Isto é, esta perspectiva ocorre em comunidades bilíngues, pois há a preservação da língua local e o inglês surge como uma língua adicional, uma vez que não existe a necessidade de impor o padrão do falante nativo.

Outra perspectiva usada para definir o uso e o ensino da Língua Inglesa na contemporaneidade é a de World Englishes (WE), que para Bolton (2004), citado por Pakir (2009), pode ser definida a partir da metáfora do guarda-chuva que se abre para todas as variedades do inglês ao redor do mundo. Ou seja, essa visão indica toda a abrangência deste idioma, isso porque tal terminologia busca abarcar todos os "novos Ingleses" que surgem em diferentes lugares do mundo sob a influência dos idiomas locais.

\section{Contexto da Pesquisa}

A presente pesquisa foi desenvolvida com doze alunos-professores do $3^{\circ}$ ano noturno do curso de Letras Estrangeiras Modernas - Inglês da Universidade Estadual de 
Londrina, ao longo do primeiro semestre de 2009 nos grupos de estudos realizados no período inicial do estágio supervisionado.

Uma das autoras do presente artigo, na condição de professora supervisora, indicava leituras as quais seriam relevantes em relação ao estágio. Nos encontros semanais, eram feitas discussões sobre as leituras com o propósito de que os alunosprofessores pudessem relacionar questões teóricas - advindas dos artigos estudados - às questões práticas - vivenciadas por eles na sala de aula de estágio ou no seu próprio contexto de atuação profissional, considerando que alguns deles já atuam no mercado de trabalho. Outra tarefa relativa aos textos e às discussões consistia na postagem de comentários em um blog elaborado pelos próprios alunos, como um meio de interação e troca de informações entre eles. Através das leituras de tais postagens, foi possível conhecer as representações que os alunos-professores elaboravam mediante a confrontação entre os pólos da teoria e da prática.

Uma das leituras realizadas foi do artigo "English in a new world language order" de Gimenez (2006), o qual apresentava uma breve discussão a respeito das diferentes terminologias acerca das concepções do que é a Língua Inglesa e seu ensino na contemporaneidade. A partir desta leitura, em um dos grupos de estudos, constatouse que era a primeira vez que a maioria dos alunos-professores tomava conhecimento de tais nomenclaturas. Sendo assim, pareceu relevante dar continuidade às leituras e discussões acerca do tema.

Posteriormente, foram sugeridas leituras adicionais, sendo estas o capítulo "Which Model?" do livro English Next de Graddol (2006) e o artigo "English as a língua franca: analyzing research frameworks in international English, world Englishes, and ELF" de Pakir (2009), juntamente com o quadro de Kachru (1989, apud MCKAY, 2002) chamado "Três círculos concêntricos do Inglês"6. Dessa forma, o trabalho desenvolvido pela supervisora de estágio e os alunos-professores foi conduzido seguindo os moldes anteriores: leitura, discussão em grupo de estudo e postagens de comentários no blog.

A fim de conhecer a compreensão que estes alunos-professores fazem dessas terminologias e, por consequência, com qual se identificam como professores de Língua Inglesa, no presente trabalho buscamos analisar as postagens feitas por estes no referido blog.

\footnotetext{
${ }^{6}$ Three concentric Circles of English.
} 


\section{Referencial teórico de análise}

Para realizar as análises dos comentários dos alunos-professores no blog utilizaremos como fundamentação teórico-metodológica a teoria da representação social, a qual tem origens na antropologia e na sociologia.

O sociólogo Émily Durkheim é o precursor de tais estudos (apud MINAYO,1995:90). Usando o termo "representações coletivas", Durkheim afirmava que tal conceito se refere às categorias de pensamento que levam determinadas sociedades a elaborar e expressar sua realidade. Essas categorias não seriam universais e nem pré-estabelecidas, mas sim constituídas através de fatos sociais e de suas transformações. Sendo assim, através da observação de tais eventos, seria possível conhecer e interpretar essa sociedade. Trata-se de compreender como os indivíduos pertencentes a um determinado grupo se apropriam da realidade e fazem sentido dela.

Os conceitos de Durkheim foram retomados e redefinidos por Moscovici (2003). Este autor usa o termo "representações sociais" o qual indica "um universo de opiniões próprias de uma cultura, de uma classe social ou um grupo, relativas aos objetos do ambiente social" (1961, p. 66). As adaptações de Moscovici surgiram no sentido de compreender sociedades modernas e toda sua dinâmica volátil, de modo que as representações sociais seriam "fenômenos específicos que estão relacionados com um modo particular de compreender e se comunicar - um modo que cria tanto a realidade como o senso comum" (MOSCOVICI, 2003, p. 49).

Considerando o contexto no qual foi desenvolvido este trabalho, temos como grupo uma coletividade composta pelos alunos-professores e supervisora de estágio, e a realidade criada - por esses indivíduos sobre terminologias acerca do ensino de Língua Inglesa na contemporaneidade - poderá ser percebida através das análises das representações expressas em seus comentários postados no blog.

Na Linguística Aplicada, Freire e Lessa (2003) definem representações sociais como:

... maneiras socialmente construídas de perceber, configurar, negociar, significar, compartilhar e/ou redimensionar fenômenos, mediadas pela linguagem e veiculadas por escolhas lexicais e/ou simbólicas expressivas que dão margem ao reconhecimento de um repertório que identifica o indivíduo e sua relação sócio-histórica com o meio, com o outro e consigo mesmo. (p.174) 
Nesse sentido compreendemos que a matéria- prima para quem busca conhecer e entender as representações que o outro tem sobre a realidade só pode ser a linguagem.

Ainda na Linguística Aplicada, o conceito de representações sociais foi revisitado por Celani e Magalhães (2003):

uma cadeia de significações, construídas nas constantes negociações entre os participantes da interação e das significações, as expectativas, as intenções, os valores e as crenças referentes a a) teorias do mundo físico; b) normas, valores e símbolos do mundo social; c) expectativas do agente sobre si mesmo como ator em um contexto particular. (p. 321)

Sendo assim, nosso foco neste artigo recai sobre o segundo item citado pelas autoras. Isso porque, por meio da análise dos comentários postados no blog pelos alunos-professores, poderemos compreender de que forma os valores e as normas referentes às diferentes terminologias em questão são por eles assimilados.

\section{Análise dos Comentários do Blog}

A fim de compreender o entendimento dos referidos alunos-professores a respeito das diferentes perspectivas acerca do uso e ensino de Língua Inglesa na contemporaneidade e com qual delas eles mais se identificam ao ensinar este idioma, analisamos os comentários que postaram no blog respondendo as seguintes perguntas: Como você compreende e identifica-se com as diferentes perspectivas sobre o ensino de língua inglesa? Qual delas você adota/adotaria?

Para melhor organizar a análise, agrupamos os comentários postados no blog em três diferentes categorias, mediante as semelhanças entre as respostas. Dessa forma, apresentamos o seguinte quadro de acordo com o que os alunos declaram identificar-se:

\begin{tabular}{|c|c|c|}
\hline $\begin{array}{c}\text { Inglês como Língua } \\
\text { Franca }\end{array}$ & $\begin{array}{c}\text { Aglutinações de } \\
\text { diferentes perspectivas }\end{array}$ & $\begin{array}{c}\text { Nenhuma das } \\
\text { perspectivas } \\
\text { apresentadas }\end{array}$ \\
\hline aluna - professora A & aluna - professora C & aluna -professora G \\
aluna- professora B & aluna - professora F \\
aluna - professora D & aluna - professora I \\
aluno - professor E & aluno - professor J & \\
& PASSONI, D'ALMAS \& AUDI
\end{tabular}


aluno - professor $\mathrm{H}$

Quadro 1: categorias de análise dos comentários postados pelos alunos-professores no blog

Devemos informar que são doze os alunos matriculados na disciplina, mas apenas dez deles postaram comentários no blog, os quais constituem os dados a serem analisados. Salientamos que a composição das categorias de análise é decorrente da análise individual das respostas dos alunos, as quais destacam, na maior parte a recorrência de uma identificação com o ensino de Inglês como Língua Franca. As demais perspectivas de ensino e uso foram mencionadas de forma associada umas às outras, sendo descritas na segunda categoria do quadro.

\subsection{Alunos-professores que se identificam com Inglês como Língua Franca:}

Nesta categoria analisaremos os comentários dos alunos-professores que declaram identificar-se com a perspectiva de ensino e uso de Inglês como Língua Franca. Porém, destacamos que, o fato de se identificarem com esta perspectiva não implica necessariamente a atuação daqueles que já estão trabalhando como professores, sendo que suas razões serão apresentadas e analisadas logo adiante. Dessa forma, nesta categoria será possível encontrarmos, em alguns dos comentários, o modo como estes alunos-professores concebem outras perspectivas que não só ELF.

A aluna-professora A é a primeira a postar um comentário no blog, dizendo que em seu contexto de trabalho atual, uma escola privada, ela ensina inglês como Língua Internacional, embora sua inclinação pessoal seja para ELF. parece relevante notar o uso do advérbio "therefore" ao apresentar qual Inglês é ensinado na escola mencionada. A parece compreender que o ensino de EIL é algo inerente às escolas privadas e que o professor nesta realidade não tem autonomia para colocar em prática as suas crenças, pois se estivesse trabalhando por conta própria, adotaria uma terminologia que considera mais adequada, como o ELF. Porém, ao justificar sua escolha, A ainda aponta que tal perspectiva parece ser útil apenas no que se refere ao ensino de pronúncia e que para ela seria difícil pensar em um falante que cometesse erros de outra natureza.

Tais representações nos levam a pensar nas definições presentes nos artigos lidos, as quais indicavam que, ao adotarmos ELF, estaremos nos distanciando do ideal do falante nativo, o que, para a aluna-professora A parece caracterizar a perspectiva 
apenas pela aceitação de diferentes pronúncias. Para ela, neste aspecto ainda é necessário haver uma língua-padrão. A aluna-professora A demonstra também partilhar de uma preocupação recorrente no processo de ensino/aprendizagem, que se refere à dificuldade em definir o que é erro dentro dessa abordagem. Ao encerrar o comentário, ela afirma que o mais importante é a comunicação, porém não fica explicitado de que forma ela definiria esta habilidade.

A: "Nowadays, I work in a private context, therefore, I have to adopt this terminology of International English, but I think if I would work by myself, I would adopt the terminology about English as a Lingua Franca. (...)But I guess to adopt this concept of ELF just work in case of accent. It is difficult to think in a speaker who speaks, but makes some kinds of mistakes, even if they are relevant. Of course the most important is the communication." 7

No seguinte comentário, a aluna-professora B informa que, assim como a alunaprofessora A, ela trabalha em contex to privado e como conseqüência disso deve adotar a variedade do Inglês britânico, que é a visão da escola. Devemos ressaltar que nem nas discussões e nem nas leituras realizadas havia esta definição acerca da Língua Inglesa, porém podemos compreender que para A escola em questão, o ensino é pautado pela visão de EFL, uma vez que considera determinada nacionalidade para denominá-lo.

Mas em sua concepção pessoal, B acredita que deveríamos escolher ELF para ministrar nossas aulas, dadas as limnitações encontradas, a seu ver, para se atingir o padrão do nativo. Devemos ressaltar o uso da primeira pessoa do plural "we", ou seja, para ela o ELF é uma abordagem que deveria ser seguida por todos os professores, sem que se explicitem, porém, quais características desta perspectiva são importantes para ela. pode-se inferir, todavia, que uma das caracterísitcas teria a ver com a falta de compreensão do contexto nativo, já que ela justifica a necessidade de se usar ELF numa situação desse tipo. B ainda ressalta que, atualmente, os alunos que procuram aprender o idioma em questão ainda veem o seu ensino como o de uma língua estrangeira. Por essa razão, torna-se complicado escolher que modelo utilizar para desenvolver atividades de ensino do idioma em questão. Assim compreendemos que a língua é uma mercadoria, e o aluno, visto como consumidor, escolhe $\mathrm{O}$ tipo ou variedade linguística que deseja comprar, ou seja, aprender.

B: "I' $m$ in the same situation as A, working in a private school which teaches just British English. I think that even if we try to speak like natives it's impossible to

\footnotetext{
${ }^{7}$ Todos os comentários retirados do blog são reproduzidos neste trabalho de forma fidedigna. 
reach perfection as in the speech as in the way we understand the language. We will not always understand the context to try to explain the language as it is understood for natives, so we'll have to use ELF. At the same time, instead to learn ELF, students are interested in learn EFL, so I think it's a bit confuse to us which model to adopt."

Quanto aos comentários da aluna-professora D, parece ainda complicado para ela entender todas as terminologias (essa 'complicação' não está evidenciada nos dados citados. seria uma inteerpretação do/a autor/a?). Ela trabalha em um instituto de idiomas no qual deve ensinar EFL pelo fato de que os alunos dela parecem ter contato com países do exterior. Esta justificativa pode nos indicar que estes alunos pertencem a um grupo social de poder aquisitivo mais elevado que a maioria da população, pois estudam num instituto privado, já viajaram ou viajarão para o exterior, têm familiares nestes países. Uma dúvida que surge do dado é a quais países a aluna-professora D está se referindo, pois ela não os nomeia. Assim, podemos inferir que pode não se tratar apenas de países onde o inglês é língua materna, ou seja, para D a língua inglesa parece ser facilitadora no acesso a diferentes países, não só aqueles do Círculo Interno, daí a possível complicação de d na compreensão das terminologias aludida no início do parágrafo :

D: "On the Institute where I "try" to develop my students proficiency on the language the method adopted is based on Teaching English as Foreign Language due to the fact that most part o them had already been abroad, have relatives or will travel soon for some reason; so the language does not seem to be so far from them."

Entretanto, a aluna-professora D continua seu comentário mencionando que adotaria ELF para o contexto de escola pública, explicando que assim o idioma poderia fazer parte da vida do aluno, de sua realidade. Ao pensar na possibilidade de adotar essa abordagem na escola pública, a aluna-professora não seria tão rigorosa em relação a erros, pois neste contexto o mais importante parece ser o fato de a pessoa ser compreendida. Ao contrastarmos as duas visões apresentadas pela mesma alunaprofessora, podemos compreender que ela associa a abordagem dada ao ensino da língua com a realidade vivida pelo aluno. Neste caso, principalmente pelo fato de mencionar a possibilidade dos alunos do contexto privado de viajar para o exterior, entendemos que esta realidade pode se pautar essencialmente pela classe social na qual o aprendiz se insere.

D:"But, If I were to choose how to teach my students in a public context, I would adopt ELF, trying to make the language part of their lives, bring it to their realities, and not taking into account some mistakes, as the goal is to make their English understandable." 
já no comentário do aluno-professor E percebemos que ele considera importante aprender sobre as terminologias utilizadas para explicar o ensino de Inglês até mesmo para que diferentes variedades do idioma sejam aceitas mais facilmente. E evidencia as características que compreende como importantes sobre a adoção do ELF, como não ter que se preocupar em soar como nativo e que seria opcional ter conhecimento sobre aspectos culturais dos países onde o inglês é língua materna. Para ele o que se destacou nos artigos lidos foi a questão da inteligibilidade relacionada ao uso de ELF, e que seria perfeito se ela puder ser alcançada.

E: "It is a good thing to be familiar with all these terms and know that in some situations the required English is the native-like, for example for social inclusion in a native ES country, but I believe that with all these discussions about ELF the things can be changed and the varieties accepted more easily. It is important to consider English as a lingua franca because people don't have to worry about native English speakers' way and do not have to know their culture, just if they want. In general English would be seen as a neutral language used just for communication, not to implicitly impose inner countries culture." As I read in the articles since there is intelligibility ELF is perfect, it neither influences nor disrespect non-native speaking countries' culture."

O aluno-professor $\mathrm{H}$, por sua vez, diz que será adepto do ELF quando entrar em sala de aula. É relevante notar a característica enfatizada por ele nesta abordagem, que seria dar noções aos alunos sobre as diferenças entre tipos de Inglês. Ou seja, o alunoprofessor parece estar preocupado em ensinar não apenas a língua, mas também instaurar neles uma consciência linguística, pois para ele seria quase impossível que todos falassem o mesmo Inglês-padrão. Porém, ao continuar o seu comentário e justificar sua escolha, $\mathrm{H}$ parece entrar em contradição, pois diz que no contexto que lecionar, irá adotar um padrão do idioma que deve ser o mais correto e adequado para o contexto. Isto é, aqui percebemos como a adoção ou não de um padrão de Inglês para o ensino é algo conflitante, o aluno-professor $\mathrm{H}$ demonstra que ainda não consegue explicar exatamente de que forma se daria o ensino pela perspectiva do ELF.

$\mathrm{H}$ : "And when I give class I will use "English as lingua franca", passing for the student notions, for that they know the differences of each English, and have a capacity of understanding and communication with any English speaker, because it would be almost impossible that all spoke an only one and standardized English, passing over the particularities of each country, but I will try to maintain a pattern that, in agreement with context that I am working, be the most correct."

\subsection{Alunos-professores que se identificam com aglutinações de diferentes} perspectivas 
Ao serem apresentados a diferentes perspectivas de ensino e uso da Língua Inglesa, muitos dos alunos-professores parecem ter compreendido que cada uma delas poder ser útil de diferentes formas, por isso não se declararam adeptos de apenas uma das terminologias. Isso fica evidente nos comentários a seguir.

A aluna-professora C, por exemplo, define com suas próprias palavras sua compreensão sobre três paradigmas de ensino/aprendizagem de Inglês, os quais parecem ser os mais relevantes para ela. Ao explicar com qual desses ela se identifica, $\mathrm{C}$ relata que, como professora de escolas privadas e institutos de idiomas, adota características de cada uma das três abordagens no que se refere a diferentes aspectos do ensino. Por adotar um padrão, se vincula ao EIL; identifica-se com WE por apresentar aos seus alunos diferentes culturas e pronúncias; e considera ELF muito importante, pois prepara seus alunos para estarem aptos a se comunicar com qualquer falante do Inglês. Tal postura pode parecer contraditória, pois adotar apenas um dos procedimentos que caracteriza cada terminologia não significaria que o ensino de Língua Inglesa ocorra pela mescla de todos esses vieses. Porém, para $\mathrm{C}$ parece não haver incompatibilidade em aglutinar essas diferentes abordagens. Através de sua fala, é possível compreendermos sua preocupação em apresentar a diversidade aos seus alunos, sem que isso influencie de fato o modo como eles irão aprender a língua como sistema. Isto é, para ela é importante que o aprendiz seja consciente a respeito da existência dos “diferentes ingleses”. Porém, ele deve dominar aquele que é o 'padrão'. Ao encerrar seu comentário, $\mathrm{C}$ afirma a necessidade de termos uma língua com a qual todos possam se comunicar, isto é, a aluna-professora pode estar conferindo um caráter mais global ao idioma.

C: "We, teachers and/or students, are facing a paradigm between three different points of view related to ELT: International English (IE), World 'Englishes' (WE), and English as a Lingua Franca (ELF). IE consists in a variety of English used in formal contexts and basically, it is used in education and teaching, considering English as Foreign Language. WE is associated to the varieties of English culture and identity, and eventually ELF, where English is a communicative tool between countries, native or not. Considering the private schools and language courses, which I am engaged teaching English, I adopt a standard English to teach, with some focus on the sociolinguistic reality of the countries where English is the official language, and also preparing students for a 'possible' contact with the language in order to communicate. The terminologies are not well defined in my posture as a teacher: adopting IE to carry on a Standard English, WE to introduce different culture and pronunciation to the students, and ELF, preparing them to communicate in English with English speakers, what they can do with other Brazilians, Americans, and a Japanese, who doesn't speak Portuguese, but speaks English. I consider essentially important ELF view nowadays. Globalization, 
business and social life need a common language to go ahead, and the world must use a language that everyone could speak, as a second language or not, but in order to follow the international rhythm."

A aluna-professora F, por sua vez, acredita que tanto o professor quanto o aluno se beneficiariam pelo contato com as diferentes terminologias e suas diferenças. Isto é, F entende que a pluralidade parece ser benéfica, as diferentes abordagens deveriam ser combinadas pelo professor em sala de aula, pois segundo ela, tal modo de ensinar Inglês ajudaria o professor a ser mais eficaz. Para esta aluna-professora cada abordagem tem peculiaridades úteis para que o aluno consiga seu objetivo, objetivo esse que para ela seria comunicação. Porém, não tomamos conhecimento sobre o que $\mathrm{F}$ entende exatamente por comunicação. sua postura de mesclagem de abordagens, na verdade, é por ela justificada pelo contexto em que vivemos, pelo mundo globalizado, no qual devemos estar preparados para mudanças, ou seja, F associa o ensino e a aprendizagem de Inglês à globalização e acredita, por essa razão, que a adoção de apenas uma das abordagens seria uma limitação.

Podemos interpretar que F está preocupada com um ensino que seja eficiente e que realmente prepare o aluno para ser um usuário competente da língua, reconhecendo seu caráter mais global. Apesar disso, não conseguimos identificar ao certo de que forma $\mathrm{F}$ acredita que esse ensino possa ocorrer.

\footnotetext{
F: "In my opinion, I believe that nowadays we should not only think about teaching and learning English in a single concept, but we should know that we are living in a Globalized World and we need to be opened and receptive for changes. These changes seem sometimes not too relevant, but they can make a lot of difference in the future of a student. Having contact with teaching terminologies such as ELF, $E F L, W E, I E$, etc..., can help us to be more effective in teaching the language because each one of them have some particularities that can help the student to achieve his aim - communication. Teachers, who have the opportunity of using more than one kind of teaching, will be able to lead the students to achieve their aims quickly and efficiently than others that do not use them."
}

No que concerne os dados da aluna-professora I, ela acredita que é possível ensinar ELF e IE concomitantemente. Isso porque um aspecto de cada uma destas abordagens parece de extrema relevância para ela: a tolerância aos erros dos aprendizes, vinculado ao ELF, e a adoção de um padrão da língua, associada ao EIL, visão essa que pode parecer ambígua. As representações desta aluna-professora indicam preocupação a respeito da necessidade de uma mudança de postura no ensino de língua, mais 
especificamente ao que se refere à tolerância dos erros durante o processo de aprendizagem o idioma em questão:

I:"I believe when we are teaching English, we can use English as a lingua franca and as an International English, because we need to teach using bases to write, read and speak, but we need some concessions, considering that our students are learning a new language and so many times can make mistakes about the correct form to use the language. It is necessary to be flexible when we are teaching, but we just can't forget about the correct norms to use grammar and have good writing and a good communication, that's why I believe that both forms to learn can be very interesting to help us in our job as teachers."

Os dados do aluno-professor $\mathrm{J}$ indicam que ele faz uma interessante analogia entre o Inglês e o Latim, o que pode indicar que ele reconhece a existência dos WE. Para $\mathrm{J}$, conhecer as diferentes perspectivas de uso e ensino do inglês parece ser relevante para o professor, a fim de que ele esteja informado sobre o que está acontecendo com o idioma em questão. Porém, $\mathbf{J}$ silencia a respeito dos alunos e das implicações que o conhecimento do professor sobre tais variações do Inglês trazem tanto para seu ensino quanto para a aprendizagem dos alunos.

$\mathrm{J}:$ "Both classical and vulgar forms of Latin are usually taken into consideration why wouldn't it be the same with English? What teacher of Latin doesn't know the two forms? One way or another, we must be prepared to deal with all these approaches. In order to be well informed about what is going on, knowing all these terms (ELF, WE, IE etc.) could be a start. I would like to teach at least two of these classes - surely, the IE would be one of them."

\subsection{Alunos-professores que não se identificam com nenhuma das perspectivas apresentadas}

De todos os alunos-professores que postaram comentários no blog sobre as diferentes concepções acerca do Inglês, e sobre qual das perspectivas a elas ligadas adotariam, a aluna-professora $\mathrm{G}$ foi a única pessoa que demonstrou não se identificar com nenhuma daquelas que foram discutidas. $\mathrm{G}$ acredita que entender todas essas perspectivas é muito importante para um bom professor de Inglês. Ela relata que leciona inglês para crianças, e por esse fato se identifica com English for Young Learners.

G: "Understanding all these terminologies and know when, where and how to take advantage of this to improve your class is the ideal of a good teacher. I'm trying to be familiar with these terms, but it's not easy. In my context (children of 5 and 6 years old)I think that could be use 'English for young learners'. The children learned by games, songs ,tales, doing crafts, respecting they rhythm." 
Essa abordagem não estava em pauta nas discussões, porém um dos textos lidos pelos alunos (Graddol, 2006) apresentava esta perspectiva de ensino de inglês. Optamos por não incluir esta abordagem na discussão pelo fato de entendermos o TEFYL muito mais como uma metodologia de ensino direcionada a uma determinada faixa etária do que uma perspectiva de ensino vinculada ao uso do Inglês.

\section{Considerações Finais}

Após a análise dos comentários postados no blog foi possível compreender que a discussão sobre as diferentes terminologias acerca do uso e do ensino de Língua Inglesa demonstra-se ainda imatura. Ou seja, alunos-professores do $3^{\circ}$ ano do curso de Letras Estrangeiras Modernas - Inglês estão tendo seu primeiro contato com o tema ao mesmo tempo em que começam a desenvolver atividades de ensino em seus estágios ou já estão atuando profissionalmente como professores em escolas privadas.

Outra questão que nos chama atenção é sobre quem ou o que determina a abordagem a ser adotada acerca da concepção e o ensino da Língua Inglesa. As alunasprofessoras que já lecionam em escolas privadas relatam ter, pessoalmente, concepções divergentes em relação ao que as instituições determinam. Há também relato de situações em que os próprios estudantes determinam que Inglês querem estudar. Diante de tais evidências, concluímos que, por mais que os cursos de formação busquem incitar a autonomia do professor, a fim de propiciar uma formação voltada para reconhecimento da diversidade, e ofereçam espaço para discussões acerca das concepções sobre o Inglês, este já se transformou em uma commodity (Jordão, 2008) na sociedade de capital. Ou seja, o trabalho do professor passa a ser alienado, ele "produz" o que o mercado consumidor quer, o que os proprietários dos meios de produção - no caso, as escolas privadas - solicitam. Sem participar intelectualmente deste processo, o professor transforma-se em apenas um operário da sala de aula.

Também percebemos que em muitos dos comentários os alunos-professores relacionaram o ensino do ELF como o mais adequado para as escolas públicas. Das características desta perspectiva, os alunos-professores salientaram a maior tolerância aos chamados erros, a inteligibilidade, a ausência da necessidade de se imitar ou soar como um falante nativo. A ênfase nestes aspectos indica a preocupação dos alunos- 
professores em desenvolver uma consciência lingüística acerca do Inglês em suas práticas de sala de aula. Devemos destacar que na formação de professores há necessidade de se direcionar o foco para as implicações que adotar esta perspectiva - ou qualquer uma das outras - pode trazer para a sala de aula.

Os alunos-professores precisam compreender que a abordagem de ELF abrange a preparação dos alunos para não apenas o que se refere a ser um usuário competente da língua, mas também busca prepará-lo para lidar com a diversidade de falantes do Inglês, bem como saber reconhecer a complexidade decorrente desse fato.

Dentre as abordagens apresentadas aos alunos-professores, o WE foi pouco mencionado. Acreditamos que isto decorra das próprias características relacionadas a esta perspectiva, ou seja, o WE está estritamente relacionado aos diferentes países, os quais adotam um tipo de Inglês que surge de maneira bastante dinâmica, carregado de influências da língua local. Isto é, como tal fenômeno não ocorre no Brasil, os alunosprofessores devem ter dificuldade em compreender as proporções do WE no mundo. Por isso, acreditamos que os cursos de formação de professores também poderiam ser um espaço no qual os alunos-professores pudessem ter contato com o WE, para o reconhecimento da diversidade e assim estarem aptos a também propiciarem al contato aos seus alunos nos diferentes contextos escolares.

Sendo assim, parece imprescindível que essas perspectivas sejam ensinadas anteriormente à prática de ensino ou estágio supervisionado dos alunos-professores, para que, desse modo, eles compreendam de forma mais abrangente as implicações da Língua Inglesa quanto a seu uso e ensino.

Ao final de nossa análise entendemos que é de extrema relevância que nos cursos de formação de professores sejam consideradas as dimensões que desencadeiam (re)significações de aspectos determinantes para a elaboração discursiva e prática dos futuros professores, isto é, o modo como as representações sociais desses indivíduos, potencialmente irão desenvolver a agentividade e empoderamento dos mesmos.

\section{Referências}

CANAGARAJAH, S. From Babel to Pentecost: postmodern glottoscapes and the globalization of English. ANGALA, L.; BARRIOS, M.L., WILLIAMS, J. Towards the knowledge society: making EFL education relevant. Cordoba, FAAPI, 2005. p. 22-32 
CELANI, M.A.A.; MAGALHÃES, M C. Representações de professores de inglês como língua estrangeira sobre suas identidades profissionais: uma proposta de reconstrução. In: MOITA LOPES, L. P.; BASTROS, L. C. (Org.). IDENTIDADES: RECORTES MULTI E INTERDISCIPLINARES. Campinas, SP: Mercado de Letras, 2002. p. 319-37.

DEWEY, M. English as a lingua franca and globalization: an interconnected perspective. International Journal of Applied Linguistics, v. 17, n. 3, p.332-351, 2007.

FREIRE, M. M; LESSA, A.B.C. Professores de inglês da Rede Pública: suas representações, seus repertórios e nossas representações. In: BÁRBARA, L; RAMOS, R.C.G. (Org). Reflexão e ações no ensino e aprendizagem de línguas. Campinas: Mercado de Letras, 2003.

GIMENEZ. T. English in a New World Language Order. In: MACHADO, L. T.; CRISTOVÃO, V.L.L.; FURTOSO, V. (Org.). Aspectos da linguagem: considerações teórico-práticas. Londrina, UEL, 2006, p. 59-72.

GRADDOL, D. English Next. British Council. 2006.

JORDÃO, C. M. A Postcolonial Framework for Brazilian ELT teachers' Social Identities. Revista Eletrónica Matices en Lenguas Extranjeras, n. 02, Dezembro, 2008. Disponível em: <www.revistamatices.unal.edu.co>. Acesso em: 10 de Julho de 2009.

LIN, A.M.Y.; MARTIN, P. From a critical deconstruction paradigm to a critical construction paradigm: an introduction to decolonization, globalization and languagein-education policy and practice. LIN, A.M.Y.; MARTIN,P. (Ed.). Decolonisation, globalization. Clevedon: Multilingual Matter, 2005.

MCKAY, S. L. Teaching English as an international language: rethinking goals and approaches. Oxford: Oxford University Press, 2002.

MINAYO, Maria Cecília de Souza. O conceito de representações sociais dentro da Sociologia clássica. In: GUARESCHI, Pedrinho; JOVCHELOVITC, Sandra et al (Org.). Textos em representações sociais. 8.ed. Petrópolis - RJ: Vozes, 1995. p. 89 111.

MOSCOVICI, S. Representações Sociais: investigação em psicologia social. Petrópolis, RJ: Ed. Vozes, 2003.

PAKIR, A. English as a lingua franca: analyzing research frameworks in international English, world Englishes, and ELF. World Englishes, v. 28, n. 2, p. 224-235, 2009.

PENNYCOOK, A. The cultural politics of English as an international language. London: Longman, 1994, p. 1-37.

SEIDLHOFER, B. Closing a conceptual gap: the case for a description of English as a lingua franca. International Journal of Applied Linguistics, v. 11, n. 2, p.133-158, 2001. 